\title{
Periodic Energy Transport and Entropy Production in Quantum Electronics
}

\author{
María Florencia Ludovico ${ }^{1}$, Liliana Arrachea ${ }^{1}$, Michael Moskalets ${ }^{2}$ and David Sánchez ${ }^{3, *}$ \\ 1 International Center for Advanced Studies, UNSAM, Campus Miguelete, 25 de Mayo y Francia, \\ 1650 Buenos Aires, Argentina; mfludovico@gmail.com (M.F.L.); lili.arrachea@gmail.com (L.A.) \\ 2 Department of Metal and Semiconductor Physics, NTU "Kharkiv Polytechnic Institute", \\ 61002 Kharkiv, Ukraine; michael.moskalets@icloud.com \\ 3 Instituto de Física Interdisciplinar y Sistemas Complejos IFISC (UIB-CSIC), \\ E-07122 Palma de Mallorca, Spain \\ * Correspondence: david.sanchez@uib.es; Tel.: +34-971-25-9887 \\ Academic Editor: Ronnie Kosloff \\ Received: 4 October 2016; Accepted: 15 November 2016; Published: 23 November 2016
}

\begin{abstract}
The problem of time-dependent particle transport in quantum conductors is nowadays a well established topic. In contrast, the way in which energy and heat flow in mesoscopic systems subjected to dynamical drivings is a relatively new subject that cross-fertilize both fundamental developments of quantum thermodynamics and practical applications in nanoelectronics and quantum information. In this short review, we discuss from a thermodynamical perspective recent investigations on nonstationary heat and work generated in quantum systems, emphasizing open questions and unsolved issues.
\end{abstract}

Keywords: quantum thermodynamics; time-dependent heat; AC-driven quantum systems

\section{Introduction}

The rapid progress in the manipulation of small systems at submicron scales has spurred the interest in the field of quantum thermodynamics. The goals of this discipline is the understanding of the fundamentals of thermodynamics in quantum systems typically driven out of equilibrium by means of external fields. Most of the recent literature focuses on static fields and the resulting stationary transport effects (for a recent review, see [1]). However, there is a growing interest in analyzing the thermodynamic properties of quantum conductors in the presence of time-dependent potentials. In this case, dynamics is the main objective of the theory as fluxes and responses depend explicitly on time [2].

Quantum electronic devices are paradigmatic realizations of open quantum systems. Typically, we can identify in these setups a finite-size piece where a small number of electrons are confined. However, these particles are not isolated. They are in contact with leads, substrates and the electromagnetic environment, which in practice constitute macroscopic thermal and particle reservoirs. We can distinguish two main classes of time-dependent processes in these systems. One of them is related to the transient behavior after a short-time non-equilibrium perturbation, where the main issue is to understand the relaxation and thermalization processes as the system approaches the equilibrium state. The other class is the response to a periodic driving. Both classes of dynamics have received a significant interest for some years now.

From the conceptual point of view, it is quite natural to relate the energy transport in periodically-driven systems to thermodynamic machines, like heat engines and refrigerators, since these typically operate in cycles. There is a lengthy literature on nanoscale quantum thermodynamics based on two-level systems [3-7], atomic [8-11] and molecular [12-14] systems, nanomechanical [15-20] 
systems and other systems represented by harmonic oscillators [21-24]. The list of works devoted to study cyclic processes in electron systems is shorter but is growing every week. In [25] heat transport induced by adiabatic pumping is analyzed in a quantum dot driven by two AC voltages operating with a phase lag. Two interesting operational modes were identified: (i) the heat pumping against a gradient of temperature and (ii) the exchange of work between the driving forces. Heat pumping by AC driving was also investigated in the non-adiabatic regime [26] and in arrays of quantum dots in the Coulomb blockade regime [27], while in [28] the effect of the exchange of power between two driven quantum capacitors was analyzed. In [29] the exchange of power between electron currents induced by DC bias voltages and adiabatic driving forces was considered and a similar mechanism was later analyzed in the context of a pair of helical edge states coupled to a magnetic island [30]. The effect of AC driving in the efficiency of usual two-terminal thermoelectric setups was addressed in $[27,31,32]$. An extended thermoelectric framework to describe the work exchanged with the AC driving sources within the adiabatic regime, on equal footing with the exchange of charge and heat between electron reservoirs with thermal and voltage biases was proposed in [33]. Thermal transport in electron systems driven by the coupling to electromagnetic radiation has been studied in [34-37]. The related problems of heat fluctuations and the definition of effective temperatures to characterize the net heat transport in these systems were addressed in [38-48] and [49-51], respectively.

In addition to the interest in the fundamental aspects, it is of paramount importance for potential applications to discriminate which portion of the energy invested to operate these devices is amenable to be used and which one is wasted by dissipation. This distinction is at the heart of thermodynamics and is conventionally addressed in quasi-stationary processes where the system under study is very weakly coupled to the reservoirs. In quantum electronics, however, the generic situation is to have the driven structure strongly coupled to the rest of the circuit, which plays the role of the reservoir. On the other hand, we are typically interested in the generation of currents, which implies non-equilibrium situations.

In this paper, we review recent developments in dynamical quantum thermodynamics applied to nanoelectronic systems. We will discuss in detail the energy transfer in out-of-equilibrium systems coupled to external baths. The analysis is based on a phase coherent mesoscopic sample (a confined system with discrete energy levels) attached to fermionic reservoirs held at a given temperature. The energies of the sample evolve with time due to the coupling with nearby AC gate terminals. Deep inside the reservoirs, electrons relax their excess energy, and the baths can thus be considered in local thermal equilibrium. We will also consider the entropy production in the whole system and will identify the different terms arising in the redistributed energy and heat $[52,53]$. Importantly, when the energies shift slowly with time, the response is adiabatic, and an exact Joule law can be demonstrated for the time domain [52-54]. In addition, we will briefly discuss the nonadiabatic regime [55] in which case the AC frequency is larger or of the order of the inverse dwell time inside the conductor. Our analysis is completely general and does not rely on the particular approach followed to evaluate the relevant dynamical quantities, like the charge and energy fluxes and the rate of entropy production.

Dynamical transport in quantum conductors is a relatively old subject with established theoretical principles [56-59], which has been investigated with different techniques [60-63]. It is well understood that a Floquet scattering matrix approach (FSM) [64] can account for photon-assisted phenomena in nanostructures (absolute negative conductance and dynamical localization [62], single-electron emission [54,65], etc.). In the present work, we also include for completeness the details of the calculation of these quantities with the Keldysh formalism using the Floquet-Fourier representation as in $[66,67]$ and discuss the connection to the FSM formulation as in [68].

\section{Theoretical Model}

We consider that our system is a slowly-driven (adiabatically) quantum conductor (e.g., a quantum dot or an array of quantum dots) coupled to several baths (fermionic reservoirs), see Figure 1 for the two terminal case. In the adiabatic regime, it is possible to propose a thermodynamic function within 
an extended resonant level model that satisfies both the first and the second laws [52,53]. We do not make any assumption on the coupling strength between the system and the baths.

Note that the problem of defining a system and a bath in the strong coupling regime has been addressed recently [69]. Moreover, the authors of [70] claim that the extended resonant level model is unable to properly describe energy fluctuations despite the fact that the expectation value of the energy is correctly derived. On the other hand, [71] finds that an influence functional approach $[6,72]$ applied to the spin-boson model can yield exact expressions for the energy dissipation in a driven open quantum system strongly coupled to a heat bath.

Here, we take into account the Hamiltonian of the fermionic baths, $\mathcal{H}_{\text {res }}=\sum_{\alpha} \mathcal{H}_{\alpha}$ with $\mathcal{H}_{\alpha}=\sum_{k_{\alpha}} \varepsilon_{k_{\alpha}} c_{k_{\alpha}}^{\dagger} c_{k_{\alpha}}$, where $\varepsilon_{k_{\alpha}}$ is the energy dispersion relation and $c_{k_{\alpha}}^{\dagger}\left(c_{k_{\alpha}}\right)$ creates (destroys) an electron with wavenumber $k_{\alpha}$, the Hamiltonian of the system, $\mathcal{H}_{S}(\mathbf{V}(t))$, where the functions $\mathbf{V}(t)=\mathbf{V}(t+\tau)=\left(V_{1}(t), \ldots, V_{M}(t)\right)$ describe the AC driving power sources capacitively coupled to the system, $\tau$ being the AC period, and the Hamiltonian of the contact region between the system and the baths, $\mathcal{H}_{\text {cont }}=\sum_{\alpha} \mathcal{H}_{c \alpha}$ with $\mathcal{H}_{c \alpha}=\sum_{k_{\alpha}}\left(w_{k_{\alpha}} c_{k_{\alpha}}^{\dagger} d_{l_{\alpha}}+\right.$ H.c $)$, where $w_{k_{\alpha}}$ are tunneling amplitudes, $l_{\alpha}$ denotes the site of the central part to which the reservoir $\alpha$ is attached, and $d_{l_{\alpha}}$, and $d_{l_{\alpha}}^{+}$destroys and creates, respectively, an electron in the central system. The three contributions add up to the Hamiltonian of the full system,

$$
\mathcal{H}(t)=\mathcal{H}_{\text {res }}+\mathcal{H}_{S}(t)+\mathcal{H}_{\text {cont }},
$$

which explicitly depends on time due to the applied AC potentials. We stress that the explicit form of $\mathcal{H}_{S}$ remains unspecified, because we will below present a thermodynamic discussion that is completely general and independent of the sample details. In fact, our presentation is also valid for interacting electrons provided these carriers are confined to the mesoscopic region. The fermionic baths are metallic leads with good screening properties and electron-electron interactions can be safely neglected there. The latter is an important remark since a gauge-invariant transport theory in the AC regime must include the self-consistent Coulomb interaction [73]. This requirement in the case of the heat current was discussed in [74].

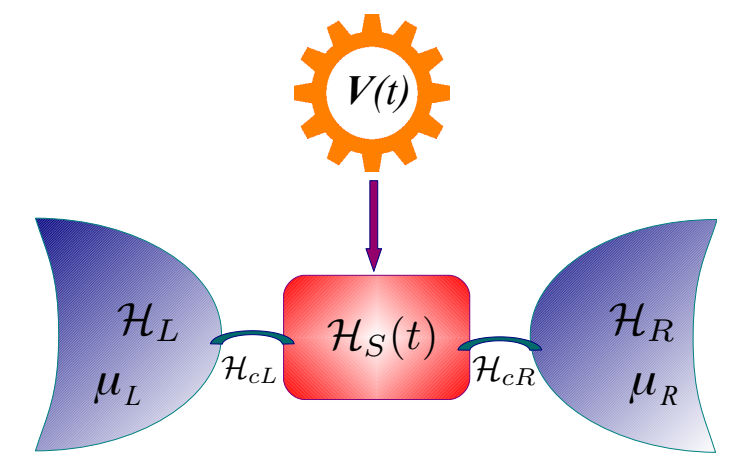

Figure 1. Sketch of the system under consideration. A quantum conductor (described by the Hamiltonian $\left.H_{S}\right)$, is coupled to two reservoirs $\left(H_{L}\right.$ and $\left.H_{R}\right)$ kept at the same temperature $T$, but with different chemical potentials $\mu_{L}$ and $\mu_{R}$. The conductor is also driven out of equilibrium by the application of AC local power sources, which are all collected in the vector $\mathbf{V}(t)$. The Hamiltonians representing the left and right contact regions are $H_{c L}$ and $H_{c R}$, respectively.

Detailed knowledge of the heat dynamics in quantum electronics is also relevant for time-dependent thermoelectrics, where temperatures may be modulated with time. A calculation for quantum $R C$ circuits shows [75] that thermoelectric charge relaxation resistance significantly differs from its purely electric counterpart. In a different setup, the thermoelectric power factor has been 
experimentally demonstrated to grow in the photo-induced regime [76]. When the coupling between a dot and its attached leads quickly changes with time, transient currents are predicted to occur in the absence of tunneling [31]. These findings call for a unified description of thermoelectrics and heat for AC-driven conductors. This is accomplished in [33], where the authors generalize the Onsager reciprocity relations for the case of adiabatic drivings. Thermodynamic forces and fluxes can be identified this way [77]. Unfortunately, experimental measurements of thermoelectric responses in the time domain are scarce. An exception is [78], where a frequency-resolved impedance spectroscopy method is used in thermoelectric modules.

\section{First Law}

Let $\rho=e^{-\beta(\mathcal{H}-\mu \mathcal{N})} / Z$ be the density matrix, with $Z=\operatorname{Tr}\left[e^{-\beta(\mathcal{H}-\mu \mathcal{N})}\right]$ the partition function, $\mathcal{N}$ the particle number and $\beta=1 /\left(k_{B} T\right)$. We start by considering $\mu_{L}=\mu=R=\mu$ and $T_{L}=T_{R}=T$. Variations $\delta \rho=\rho(t+\delta t)-\rho(t)$ are due to changes in $\delta \mathbf{V}=\mathbf{V}(t+\delta t)-\mathbf{V}(t)$, which we assume to be small. Then, both the particle number and internal energy change according to:

$$
\delta N_{v}=\operatorname{Tr}\left[\delta \rho \mathcal{N}_{v}\right], v=\alpha, S
$$

and:

$$
\delta U_{v}=\operatorname{Tr}\left[\delta \rho \mathcal{H}_{v}\right], v=\alpha, c \alpha, S
$$

respectively. The particle number and energy for the full system remain constant, since the system plus the baths can be considered as a larger, closed system.

$$
\delta N=\sum_{\alpha} \delta N_{\alpha}+\delta N_{S}
$$

and

$$
\delta U=\operatorname{Tr}[\delta \rho \mathcal{H}]=\sum_{\alpha}\left[\delta U_{\alpha}+\delta U_{c \alpha}\right]+\delta U_{S},
$$

The first law of thermodynamics states that total heat in the system is:

$$
\left.\delta Q_{\text {tot }}\right|_{\mu, T}=\sum_{\alpha}\left[\delta U_{\alpha}+\delta U_{c \alpha}-\mu \delta N_{\alpha}\right]+\delta U_{S}-\delta W_{\mathrm{AC}}-\mu \delta N_{S},
$$

where:

$$
\delta W_{\mathrm{AC}}=-\operatorname{Tr}[\rho \partial \mathcal{H} / \partial \mathbf{V}] \delta \mathbf{V}
$$

is the work developed by the time-dependent driving sources. The corresponding change in the entropy reads:

$$
\left.T \delta S\right|_{\mu, T}=\left.\delta Q_{\text {tot }}\right|_{\mu, T}=-\delta W_{\mathrm{AC}},
$$

where we have used in the last equality the conservation of particles and energy expressed by Equations (2) and (3).

We now consider a more general situation, where the reservoirs have slightly different chemical potentials $\mu_{\alpha}=\mu+\delta \mu_{\alpha}$. This implies investing an extra electric work done by DC bias voltages

$$
\delta W_{\mathrm{el}}=\sum_{\alpha} \delta \mu_{\alpha} \delta N_{\alpha}
$$

Here, $\delta \mu_{\alpha}$ is a small departure of the electrochemical potential of lead $\alpha$ with respect to the common chemical potential $\mu$.

Equation (8) generalizes to:

$$
\left.T \delta S\right|_{\mu, T}+\left.T \delta S\right|_{\delta \mu}=\delta Q_{\text {tot }}
$$


with

$$
\left.T \delta S\right|_{\delta \mu}=-\sum_{\alpha} \delta \mu_{\alpha} \delta N_{\alpha}=-\delta W_{\mathrm{el}} .
$$

Since $\delta N=\delta U=0$ due to particle and energy conservation, the first law implies that the total heat generated in the full system stems from the work done by the AC and the DC sources,

$$
\delta Q_{\mathrm{tot}}=-\delta W_{\mathrm{AC}}-\delta W_{\mathrm{el}}
$$

\section{Heat Current and Power}

The rate of change for the energy corresponding to the different parts of the system (sample $S$, reservoir $\alpha$ or coupling region $c \alpha$ ) is given by the exact quantum-mechanical expression

$$
J_{v}^{E}(t)=\frac{i}{\hbar}\left\langle\left[\mathcal{H}, \mathcal{H}_{v}\right]\right\rangle,
$$

where $v \equiv S, \alpha, c \alpha$. The time variation of the expected value of the total Hamiltonian $\mathcal{H}$,

$$
\langle\dot{\mathcal{H}}\rangle=\sum_{\alpha}\left[J_{\alpha}^{E}(t)+J_{c \alpha}^{E}(t)\right]+J_{S}^{E}(t)-\mathbf{F} \cdot \dot{\mathbf{V}},
$$

is given by the explicit time derivative of $\langle\mathcal{H}\rangle$ in terms of the generalized force $\mathbf{F}=-\langle\partial \mathcal{H} / \partial \mathbf{V}\rangle$. On the other hand, we know that the change in time of the total energy stored in the full setup, containing the central system, contacts and reservoirs, is equal to the total power developed by the external AC sources, $\langle\dot{\mathcal{H}}\rangle=-P_{\mathrm{AC}}(t)$. The latter reads:

$$
P_{\mathrm{AC}}(t)=\mathbf{F} \cdot \dot{\mathbf{V}} \text {. }
$$

Hence, it follows that the fluxes obey:

$$
\sum_{\alpha}\left[J_{\alpha}^{E}(t)+J_{\mathcal{C} \alpha}^{E}(t)\right]+J_{S}^{E}(t)=0 .
$$

The time variation of the charges present in the different pieces of the system can be similarly inferred. We start from:

$$
I_{v}^{C}(t)=e\left\langle\dot{\mathcal{N}}_{v}\right\rangle=\frac{i e}{\hbar}\left\langle\left[\mathcal{H}, \mathcal{N}_{v}\right]\right\rangle,
$$

with $v=\alpha$, S. Conservation of the total charge demands that the currents fulfill:

$$
e\langle\dot{\mathcal{N}}\rangle=I_{S}^{C}(t)+\sum_{\alpha} I_{\alpha}^{C}(t)=0
$$

Noticeably, the coupling region contributes to the energy current balance (Equation (16)) but not to its particle counterpart (Equation (18)). This will have important consequences for the heat current definition, as explained below.

Using Equation (6) it is straightforward to derive the total heat flux,

$$
\begin{aligned}
\dot{Q}_{\text {tot }}(t)= & \sum_{\alpha}\left[J_{\alpha}^{E}(t)-\mu_{\alpha} \frac{I_{\alpha}^{C}(t)}{e}+J_{c \alpha}^{E}(t)\right]+J_{S}^{E}(t) \\
& -P_{\mathrm{AC}}(t)-\mu \frac{I_{S}^{C}(t)}{e} .
\end{aligned}
$$

Now, we define:

$$
P_{\mathrm{el}}(t)=\sum_{\alpha} \delta \mu_{\alpha} I_{\alpha}^{C}(t) / e,
$$


which is the electric power developed by the batteries applied to the fermionic baths to keep the electron flow. It follows from Equations (16) and (18) that

$$
\dot{Q}_{\text {tot }}(t)=-P_{\mathrm{AC}}(t)-P_{\mathrm{el}}(t)
$$

which states that the total heat flux in the system is produced by the AC and DC driving powers, as expected.

The question now is how to define physically-meaningful time-dependent currents flowing through different pieces of the setup. The work in [52] demonstrates that the conventional definition for the heat flowing in the reservoir $\alpha$,

$$
\dot{\tilde{Q}}_{\alpha}(t)=J_{\alpha}^{E}(t)-\mu_{\alpha} \frac{I_{\alpha}^{C}(t)}{e},
$$

suffers from serious drawbacks. In particular, for a slowly-driven quantum level coupled to a single reservoir the use of $\dot{\tilde{Q}}$ leads to negative heat fluxes at zero temperature. This would imply that heat would be extracted from a reservoir at zero temperature, which makes no sense if we are to interpret this heat as dissipation. In contrast, when one considers the expression

$$
\dot{Q}_{\alpha}(t)=J_{\alpha}^{E}(t)+\frac{J_{\mathcal{C} \alpha}^{E}(t)}{2}-\mu_{\alpha} \frac{I_{\alpha}^{C}(t)}{e} .
$$

one finds that the heat flux is always positive at zero temperature, i.e., energy enters into the reservoir and is evacuated from the power source, as should be. Moreover, Equation (23) is a sound expression that fulfills four nice properties:

- It leads at low frequencies to a correct Joule law valid for all times [52].

- It shows perfect agreement between the Green function approach and the scattering matrix formalism [52].

- It displays parity symmetry upon reversal of the $\mathrm{AC}$ frequency even for interacting quantum conductors [79].

- It reduces to the conventional definition in the stationary case since the term $J_{c \alpha}^{E}(t) / 2$ vanishes after time averaging [53].

However, $[80,81]$ propose not to use any heat flux definition due to apparent contradictions with the third law of thermodynamics and the onset of thermodynamic inconsistencies. We would like to clarify and highlight that those references consider a very different setup, in which the time dependence of the Hamiltonian also takes place in the contact region (in our case only the central part of the system evolves in time). This might seem as a minor difference, but on the contrary it is a fundamental point, because in that case there is a part of the energy stored in the contact region that should be interpreted as dissipative power and hence it will not contribute to the heat flux.

We emphasize that the extra term $J_{\mathcal{C} \alpha}^{E}(t) / 2$ has a pure dynamical origin, since it vanishes when it is averaged over a period. This makes sense from the physical point of view, because in a period the energy can be stored only at the reservoirs. On the other hand, this contribution of the contact region is also unique to the heat current. For example, the particle flux is independent of the charge variation in the coupling region, simply because it does no exist. In stark contrast, the tunneling term does contribute to the energy transfer between the quantum system and the bath and must be taken into account in any thermodynamically consistent calculation of time-dependent heat currents. Analogously, we can define the heat variation in the sample as

$$
\dot{Q}_{S}(t)=\dot{E}_{S}(t)-\mu \frac{I_{S}^{C}(t)}{e}+\sum_{\alpha} \frac{J_{c \alpha}^{E}(t)}{2} .
$$

such that the total heat satisfies $\dot{Q}_{\text {tot }}(t)=\sum_{\alpha} \dot{Q}_{\alpha}(t)+\dot{Q}_{S}(t)$, see Figure 2 . 


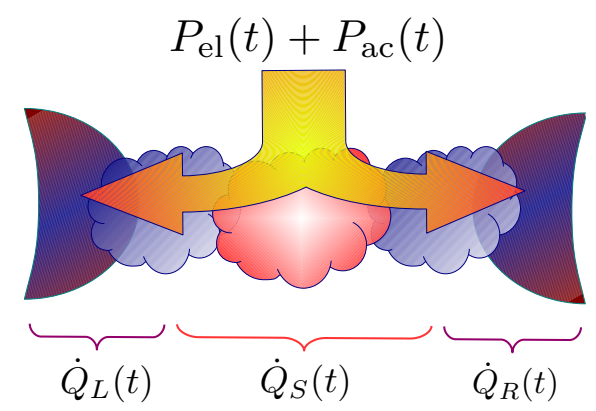

Figure 2. The total heat flux in the system is produced by the AC and DC driving sources, which is summarized by the relation $\dot{Q}_{\text {tot }}(t)=-P_{\mathrm{AC}}(t)-P_{\mathrm{el}}(t)$. The definition of the heat currents entering the reservoirs, as well as the heat variation in the central part, takes into account half of the energy stored in the contact regions. The heat flux of the central piece contributes purely dynamically, since it is zero when averaged over one period of the AC driving potentials. In the stationary state, charge and energy can be stored only at the reservoirs.

\section{Entropy Production}

Further insight can be gained if we examine the instantaneous entropy production, which is presumably a meaningful quantity for low driving frequencies, for which the system appears to be almost stationary and the classical laws of thermodynamics remain valid. Otherwise, for high enough frequencies, the nonequilibrium and quantum effects may be significant, which may hinder the use of the classical thermodynamics. This regime requires further investigation. The total entropy production has been demonstrated to follow an integral fluctuation theorem for a driven system strongly coupled to a heat bath [82]. Here, we stress that the entropy production associated with dissipative processes is always positive, in agreement with the second law. We begin by noticing that the AC forces have, in general, conservative (cons) and nonconservative (nc) components. In fact, in [33] it was shown that $\mathbf{F}(t)=\mathbf{F}_{t}+\mathbf{F}^{\mathrm{nc}}(t)$, where the first term corresponds to the Born-Oppenheimer component, which is evaluated with the equilibrium density matrix $\rho_{t}$ corresponding to the frozen Hamiltonian at the time $t$ and $\mathbf{F}^{\mathrm{nc}}(t)$ collects the nonequilibrium components. The first term corresponds to an evolution given by a sequence of equilibrium states which are, thus, reversible and do not contribute to the entropy production. Hence $P_{\mathrm{AC}}(t)=P^{\mathrm{cons}}(t)+P_{\mathrm{AC}}^{\mathrm{nc}}(t)$. The electric power of the batteries is purely non-conservative (dissipative). In this way, the total power can be split into dissipative and conservative forces $P_{\text {tot }}(t)=P_{\mathrm{el}}(t)+P_{\mathrm{AC}}(t)=P_{\text {tot }}^{\text {diss }}(t)+P^{\text {cons }}(t)$, where $P_{\text {tot }}^{\text {diss }}(t)=P_{\mathrm{el}}(t)+P_{\mathrm{AC}}^{\text {nc }}(t)$.

As a result, the total entropy production reads,

$$
\dot{S}^{\text {diss }}(t)=\frac{1}{T}\left[\sum_{\alpha} \dot{Q}_{\alpha}(t)+\dot{Q}_{S}^{\text {diss }}(t)\right]=-\frac{1}{T} P_{\text {tot }}^{\text {diss }}(t) .
$$

We have defined $\dot{Q}_{S}^{\text {diss }}(t)=\dot{Q}_{S}(t)-P^{\text {cons }}(t)$, i.e., we identify the irreversible heat production at the central system as the result of subtracting the power developed by the conservative AC forces to the total heat instantaneously stored by the electrons that are in direct contact with the AC sources. After averaging over an AC cycle, the conservative part of the heat current vanishes. Furthermore, the heat variation in the sample also vanishes, $\overline{\dot{Q}_{S}^{\text {diss }}}=\overline{\dot{Q}_{S}}=0$. The remaining terms have nonzero averages, and hence $\dot{S}^{\text {diss }}(t) \geq 0$ at all times, in agreement with the second law of thermodynamics. This is explicitly shown in [53] within an adiabatic expansion, valid for low driving frequencies. Here, we analyze an example beyond the adiabatic regime and show that $\dot{S}^{\text {diss }}(t) \geq 0$ even though for some times, the net heat flow at the reservoirs might be negative, $\sum_{\alpha} \dot{Q}_{\alpha}(t)<0$. 


\section{Calculation of the Currents within Green's Function Approach for Non Interacting Systems}

We now consider that the central part of the system is composed by a discrete chain of $N$ sites (e.g., an array of quantum dots), to which many time-periodic potentials $V_{i}(t)$ are applied. Then, the Hamiltonian of the central piece reads:

$$
H_{S}(t)=H_{S}^{0}+\sum_{m} V_{m}(t) d_{m}^{\dagger} d_{m}
$$

where

$$
H_{S}^{0}=\sum_{m=1}^{N} \varepsilon_{0}^{m} d_{m}^{\dagger} d_{m}+\sum_{m=1}^{N-1}\left(t_{m, m+1} d_{m}^{\dagger} d_{m+1}+\text { h.c. }\right)
$$

is the stationary part of the Hamiltonian, with $\varepsilon_{0}^{m}$ the energy of the site $m$ and $t_{m, m+1}$ the hopping between two consecutive sites.

\subsection{Time Resolved Charge and Energy Currents Entering the Reservoirs}

The charge and energy fluxes are defined from the expectation value of the time derivative of the number of particles and the energy of the reservoir, as in Equations (13) and (17). These currents are positive when entering the reservoir $\alpha$ and since $\left[H_{\text {res }}, N_{\alpha}\right]=\left[H_{S}, N_{\alpha}\right]=\left[H_{\text {res }}, H_{\alpha}\right]=\left[H_{S}, H_{\alpha}\right]=0$, they can be written as:

$$
I_{\alpha}(t)=\frac{i e}{\hbar}\left\langle\left[H_{c \alpha}, N_{\alpha}\right]\right\rangle=-\frac{2 e}{\hbar} \operatorname{Re}\left\{\sum_{k_{\alpha}} w_{k_{\alpha}} G_{l_{\alpha}, k_{\alpha}}^{<}(t, t)\right\},
$$

and

$$
J_{\alpha}^{E}(t)=\frac{i}{\hbar}\left\langle\left[H_{c \alpha}, H_{\alpha}\right]\right\rangle=-\frac{2}{\hbar} \operatorname{Re}\left\{\sum_{k_{\alpha}} \varepsilon_{k_{\alpha}} w_{k_{\alpha}} G_{l_{\alpha}, k_{\alpha}}^{<}(t, t)\right\},
$$

where $G_{l_{\alpha}, k_{\alpha}}^{<}(t, t) \equiv i\left\langle c^{\dagger} k_{\alpha}(t) d_{l_{\alpha}}(t)\right\rangle$ is a Green's function that involves operators of the reservoir $c^{\dagger} k_{\alpha^{\prime}}$ as well as the central part, $d_{l_{\alpha}}$. From the Dyson equation and Langreth rules [61], Green's function above can be expressed as follows

$$
G_{l_{\alpha}, k_{\alpha}}^{<}(t, t)=\int_{-\infty}^{\infty} d t_{1} w_{k_{\alpha}}^{*}\left[G_{l_{\alpha}, l_{\alpha}}^{R}\left(t, t_{1}\right) g_{k_{\alpha}}^{<}\left(t_{1}-t\right)+G_{l_{\alpha}, l_{\alpha}}^{<}\left(t, t_{1}\right) g_{k_{\alpha}}^{A}\left(t_{1}-t\right)\right],
$$

with $G_{m, n}^{R}\left(t, t^{\prime}\right)=-i \theta\left(t-t^{\prime}\right)\left\langle\left\{d_{m}(t) d_{n}^{\dagger}\left(t^{\prime}\right)\right\}\right\rangle$ and $G_{m, n}^{<}\left(t, t^{\prime}\right)=-i\left\langle\left\{d_{n}^{\dagger}\left(t^{\prime}\right) d_{m}(t)\right\}\right\rangle$ being the retarded and the lesser Green's functions of the central piece, and

$$
\begin{gathered}
g_{k_{\alpha}}^{<}\left(t-t^{\prime}\right)=i \int_{-\infty}^{\infty} \frac{d \varepsilon}{2 \pi} f_{\alpha}(\varepsilon) \rho_{k_{\alpha}}(\varepsilon) e^{-i \varepsilon\left(t-t^{\prime}\right) / \hbar}, \\
g_{k_{\alpha}}^{A}\left(t-t^{\prime}\right)=\int_{-\infty}^{\infty} \frac{d \varepsilon}{2 \pi} \int_{-\infty}^{\infty} \frac{d \varepsilon^{\prime}}{2 \pi} \frac{\rho_{k_{\alpha}}\left(\varepsilon^{\prime}\right)}{\varepsilon-\varepsilon^{\prime}-i 0^{+}} e^{-i \varepsilon\left(t-t^{\prime}\right) / \hbar},
\end{gathered}
$$

the Green's functions of the uncoupled reservoirs, with $\rho_{k_{\alpha}}(\varepsilon)=2 \pi \delta\left(\varepsilon-\varepsilon_{k_{\alpha}}\right)$, and $f_{\alpha}(\varepsilon)=$ $\left[e^{\left(\varepsilon-\mu_{\alpha}\right) /\left(k_{B} T\right)}+1\right]^{-1}$ the Fermi-Dirac distribution. Then, substituting Equations (30)-(32) in Equation (28), the charge current entering the reservoir $\alpha$ can be written as

$$
I_{\alpha}(t)=-\frac{2 e}{h} \operatorname{Re}\left\{\int d t_{1} \int d \varepsilon e^{-i \varepsilon\left(t_{1}-t\right) / \hbar}\left[i G_{l_{\alpha}, l_{\alpha}}^{R}\left(t, t_{1}\right) f_{\alpha}(\varepsilon) \Gamma_{\alpha}(\varepsilon)+G_{l_{\alpha}, l_{\alpha}}^{<}\left(t, t_{1}\right) \int \frac{d \varepsilon^{\prime}}{2 \pi} \frac{\Gamma_{\alpha}\left(\varepsilon^{\prime}\right)}{\left(\varepsilon-\varepsilon^{\prime}-i 0^{+}\right)}\right]\right\},
$$

where we have defined the hybridization as $\Gamma_{\alpha}=\sum_{k_{\alpha}}\left|w_{k_{\alpha}}\right|^{2} \rho_{k_{\alpha}}$. We now consider the Dyson equation for the lesser Green's function [68]

$$
G_{n, m}^{<}\left(t, t^{\prime}\right)=\sum_{\alpha} \int d t_{1} d t_{2} G_{n, l_{\alpha}}^{R}\left(t, t_{1}\right) \Sigma_{\alpha}^{<}\left(t_{1}-t_{2}\right)\left[G_{m, l_{\alpha}}^{R}\left(t^{\prime}, t_{2}\right)\right]^{*},
$$


with $\Sigma_{\alpha}^{<}(\varepsilon)=i f_{\alpha}(\varepsilon) \Gamma_{\alpha}(\varepsilon)$, and we introduce the Floquet-Fourier representation [66], which is convenient for periodic fields:

$$
\hat{G}^{R}\left(t, t^{\prime}\right)=\int \frac{d \varepsilon}{2 \pi} \hat{G}^{R}(t, \varepsilon) e^{-i \varepsilon\left(t-t^{\prime}\right) / \hbar},
$$

with

$$
\hat{G}^{R}(t, \varepsilon)=\sum_{n=-\infty}^{\infty} \hat{\mathcal{G}}(n, \varepsilon) e^{-i n \omega t},
$$

where $\omega=2 \pi / \tau$ is the oscillation frequency of the AC parameters $\mathbf{V}(t)$, and $\hat{\mathcal{G}}(n, \varepsilon)$ are the Floquet components. Green's function $\hat{G}^{R}(t, \varepsilon)$, can be obtained by solving the Dyson equation $[66,67]$ :

$$
\hat{G}^{R}(t, \varepsilon)=\hat{G}^{0}(\varepsilon)+\sum_{n=-\infty}^{\infty} \hat{G}^{R}(t, \varepsilon+n \hbar \omega) \hat{V}(n) \hat{G}^{0}(\varepsilon) e^{-i n \omega t},
$$

with $\hat{G}^{0}(\varepsilon)=\left[\varepsilon \hat{I}-\hat{H}_{S}^{0}-i \hat{\Gamma} / 2\right]^{-1}$ being the stationary retarded Green's function and $\hat{\Gamma}_{m, n}=$ $\sum_{\alpha=L, R} \delta_{m, n} \delta_{l_{\alpha}, m} \Gamma_{\alpha}$. The elements of the matrix $\hat{V}_{l, l^{\prime}}(n)=\delta_{l, l^{\prime}} \sum_{i} \delta_{l, i} V_{i}(n)$ are the Fourier components of $\hat{V}_{l, l^{\prime}}(t)=\sum_{n} \hat{V}_{l, l^{\prime}}(n) e^{i n \omega t}$.

Replacing Equations (35) and (36) into Equation (33), we get

$$
\begin{aligned}
I_{\alpha}(t)= & -\frac{2 e}{h} \operatorname{Re}\left\{\sum _ { l } e ^ { - i l \omega t } \int d \varepsilon \left[i \mathcal{G}_{l_{\alpha}, l_{\alpha}}(l, \varepsilon) f_{\alpha}(\varepsilon) \Gamma_{\alpha}(\varepsilon)\right.\right. \\
& \left.\left.+\sum_{\beta=L, R} \sum_{n} i \mathcal{G}_{l_{\alpha}, l_{\beta}}(l+n, \varepsilon) \mathcal{G}_{l_{\alpha}, l_{\beta}}^{*}(n, \varepsilon) f_{\beta}(\varepsilon) \Gamma_{\beta}(\varepsilon) \int \frac{d \varepsilon^{\prime}}{2 \pi} \frac{\Gamma_{\alpha}\left(\varepsilon^{\prime}\right)}{\left(\varepsilon-\left(\varepsilon^{\prime}-n \hbar \omega\right)-i 0^{+}\right)}\right]\right\} .
\end{aligned}
$$

It is convenient to express the charge current in terms of differences between the Fermi functions of the reservoirs. To this end, we first explicitly write the real part as the sum of a complex number and its conjugate

$$
\begin{aligned}
I_{\alpha}(t)= & -\frac{e}{h} \int d \varepsilon \sum_{l} i e^{-i l \omega t}\left[f_{\alpha}(\varepsilon) \Gamma_{\alpha}(\varepsilon)\left(\mathcal{G}_{l_{\alpha}, l_{\alpha}}(l, \varepsilon)-\mathcal{G}_{l_{\alpha}, l_{\alpha}}^{*}(-l, \varepsilon)\right)\right. \\
& +\sum_{\beta=L, R} \sum_{n} f_{\beta}(\varepsilon) \Gamma_{\beta}(\varepsilon) \mathcal{G}_{l_{\alpha}, l_{\beta}}(l+n, \varepsilon) \mathcal{G}_{l_{\alpha}, l_{\beta}}^{*}(l, \varepsilon) \\
& \left.\int \frac{d \varepsilon^{\prime}}{2 \pi}\left(\frac{\Gamma_{\alpha}\left(\varepsilon^{\prime}\right)}{\varepsilon-\left(\varepsilon^{\prime}-n \hbar \omega\right)-i 0^{+}}-\frac{\Gamma_{\alpha}\left(\varepsilon^{\prime}+l \hbar \omega\right)}{\varepsilon-\left(\varepsilon^{\prime}-n \hbar \omega\right)+i 0^{+}}\right)\right] .
\end{aligned}
$$

Within the wide band limit, the hybridization can be considered a constant function $\Gamma_{\alpha}(\varepsilon) \sim \Gamma_{\alpha}$. Taking into account also the following relation

$$
\frac{1}{\varepsilon-\varepsilon^{\prime}-i 0^{+}}=\mathcal{P}\left\{\frac{1}{\varepsilon-\varepsilon^{\prime}}\right\}+i \pi \delta\left(\varepsilon-\varepsilon^{\prime}\right)
$$

we find

$$
\begin{aligned}
I_{\alpha}(t)= & -\frac{e}{h} \int d \varepsilon \sum_{l} i e^{-i l \omega t} \Gamma_{\alpha}\left[f_{\alpha}(\varepsilon)\left(\mathcal{G}_{l_{\alpha}, l_{\alpha}}(l, \varepsilon)-\mathcal{G}_{l_{\alpha}, l_{\alpha}}^{*}(-l, \varepsilon)\right)\right. \\
& \left.+i \sum_{\beta=L, R} \sum_{n} f_{\beta}(\varepsilon-n \hbar \omega) \Gamma_{\beta} \mathcal{G}_{l_{\alpha}, l_{\beta}}(l+n, \varepsilon-n \hbar \omega) \mathcal{G}_{l_{\alpha}, l_{\beta}}^{*}(n, \omega-n \omega)\right] .
\end{aligned}
$$

Finally, using the identity for the spectral components [68]

$$
i \mathcal{G}_{l, l^{\prime}}(n, \varepsilon)-i \mathcal{G}_{l, l^{\prime}}^{\dagger}(-n, \varepsilon+n \omega)=\sum_{\beta=L, R} \sum_{n^{\prime}} \mathcal{G}_{l, l_{\beta}}\left(n+n^{\prime}, \varepsilon-n^{\prime} \omega\right) \Gamma_{\beta} \mathcal{G}_{l^{\prime}, l_{\beta}}^{*}\left(n^{\prime}, \varepsilon-n^{\prime} \omega\right)
$$


the charge current reads

$$
\begin{aligned}
I_{\alpha}(t)= & -\frac{e}{h} \int d \varepsilon \sum_{l} e^{-i l \omega t} \Gamma_{\alpha}\left\{i \mathcal{G}_{l_{\alpha}, l_{\alpha}}^{*}(-l, \varepsilon)\left[f_{\alpha}(\varepsilon-l \hbar \omega)-f_{\alpha}(\varepsilon)\right]\right. \\
& \left.-\sum_{\beta=L, R} \sum_{n} \Gamma_{\beta} \mathcal{G}_{l_{\alpha}, l_{\beta}}(l+n, \varepsilon-n \hbar \omega) \mathcal{G}_{l_{\alpha}, l_{\beta}}^{*}(n, \varepsilon-n \hbar \omega)\left[f_{\beta}(\varepsilon-n \hbar \omega)-f_{\alpha}(\varepsilon)\right]\right\} .
\end{aligned}
$$

Following the same procedure, we can express the energy current entering reservoir $\alpha$ in Equation (29) as

$$
J_{\alpha}^{E}(t)=-\frac{2 e}{h} \operatorname{Re}\left\{\int d t_{1} \int d \varepsilon e^{-i \varepsilon\left(t_{1}-t\right) / \hbar}\left[i G_{l_{\alpha}, l_{\alpha}}^{R}\left(t, t_{1}\right) f_{\alpha}(\varepsilon) \Gamma_{\alpha}(\varepsilon) \varepsilon+G_{l_{\alpha}, l_{\alpha}}^{<}\left(t, t_{1}\right) \int \frac{d \varepsilon^{\prime}}{2 \pi} \frac{\Gamma_{\alpha}\left(\varepsilon^{\prime}\right) \varepsilon^{\prime}}{\left(\varepsilon-\varepsilon^{\prime}-i 0^{+}\right)}\right]\right\},
$$

where we have used inside the integral that it is possible to make the replacement $\varepsilon_{k_{\alpha}} \delta\left(\varepsilon-\varepsilon_{k_{\alpha}}\right) \rightarrow$ $\varepsilon \delta\left(\varepsilon-\varepsilon_{k_{\alpha}}\right)$. Now, using the Floquet representation for Green's functions and repeating the same steps as for the charge current, we find

$$
\begin{aligned}
J_{\alpha}^{E}(t)= & -\frac{e}{h} \int d \varepsilon \sum_{l} e^{-i l \omega t} \Gamma_{\alpha}\left\{i \mathcal{G}_{l_{\alpha}, l_{\alpha}}^{*}(-l, \varepsilon)\left[(\varepsilon-l \hbar \omega) f_{\alpha}(\varepsilon-l \hbar \omega)-\varepsilon f_{\alpha}(\varepsilon)\right]\right. \\
& -\sum_{n, \beta=L, R} \sum_{n} \Gamma_{\beta} \mathcal{G}_{l_{\alpha}, l_{\beta}}(l+n, \varepsilon-n \hbar \omega) \mathcal{G}_{l_{\alpha}, l_{\beta}}^{*}(n, \varepsilon-n \hbar \omega) \\
& {\left.\left[\left(\varepsilon-\frac{l \hbar \omega}{2}\right) f_{\beta}(\varepsilon-n \hbar \omega)-\varepsilon f_{\alpha}(\varepsilon)\right]\right\} . }
\end{aligned}
$$

\subsection{Energy Stored in the Contact Regions}

The rate of change for the energy in the contact regions $J_{c \alpha}^{E}(t)=\left\langle\dot{H}_{c \alpha}\right\rangle$ can be also expressed in terms of Green's function $G_{l_{\alpha}, k_{\alpha}}^{<}(t, t)$

$$
J_{c \alpha}^{E}(t)=\sum_{k_{\alpha}}\left[w_{k_{\alpha}} \frac{d\left\langle c_{k_{\alpha}}^{\dagger}(t) d_{l_{\alpha}}(t)\right\rangle}{d t}+h . c\right]=-2 \sum_{k_{\alpha}} \operatorname{Im}\left\{w_{k_{\alpha}} \frac{d G_{l_{\alpha}, k_{\alpha}}^{<}(t, t)}{d t}\right\} .
$$

Following a similar procedure for the charge and energy currents, we find that the energy stored in the contact region reads

$$
J_{\mathcal{c} \alpha}^{E}(t)=\int \frac{d \varepsilon}{2 \pi} \omega \Gamma_{\alpha} \sum_{l} l\left[2 \operatorname{Im}\left\{e^{-i l \omega t} \mathcal{G}_{l_{\alpha}, l_{\alpha}}(l, \varepsilon)\right\} f_{\alpha}(\varepsilon)+\sum_{n} \sum_{\beta=L, R} f_{\beta}(\varepsilon) \Gamma_{\beta} \operatorname{Re}\left\{e^{-i l \omega t} \mathcal{G}_{l_{\alpha}, l_{\beta}}(l+n, \varepsilon) \mathcal{G}_{l_{\alpha}, l_{\beta}}^{*}(n, \varepsilon)\right\}\right] .
$$

It is easy to show that the second term of the above equation vanishes, since:

$$
\begin{aligned}
& \sum_{l>0} \sum_{n}\left(l \operatorname{Re}\left\{e^{-i l \omega t} \mathcal{G}_{l_{\alpha}, l_{\beta}}(l+n, \varepsilon) \mathcal{G}_{l_{\alpha}, l_{\beta}}^{*}(n, \varepsilon)\right\}-l \operatorname{Re}\left\{e^{i l \omega t} \mathcal{G}_{l_{\alpha}, l_{\beta}}(-l+n, \varepsilon) \mathcal{G}_{l_{\alpha}, l_{\beta}}^{*}(n, \varepsilon)\right\}\right) \\
= & \sum_{l>0} \sum_{n} l\left(\operatorname{Re}\left\{e^{-i l \omega t} \mathcal{G}_{l_{\alpha}, l_{\beta}}(l+n, \varepsilon) \mathcal{G}_{l_{\alpha}, l_{\beta}}^{*}(n, \varepsilon)\right\}-\operatorname{Re}\left\{e^{i l \omega t} \mathcal{G}_{l_{\alpha}, l_{\beta}}^{*}(l+n, \varepsilon) \mathcal{G}_{l_{\alpha}, l_{\beta}}(n, \varepsilon)\right\}\right)=0 .
\end{aligned}
$$

Hence,

$$
J_{\mathcal{~} \alpha}^{E}(t)=\int \frac{d \varepsilon}{h} f_{\alpha}(\varepsilon) \Gamma_{\alpha} \sum_{l} l \hbar \omega 2 \operatorname{Im}\left\{e^{-i l \omega t} \mathcal{G}_{l_{\alpha}, l_{\alpha}}(l, \varepsilon)\right\}
$$

Combining this expression with Equation (36), we find the expression:

$$
J_{c \alpha}^{E}(t)=\int \frac{d \varepsilon}{2 \pi} f_{\alpha}(\varepsilon) \Gamma_{\alpha} 2 \operatorname{Re}\left\{\partial_{t} G_{l_{\alpha}, l_{\alpha}}^{R}(t, \varepsilon)\right\},
$$


which clearly shows that this energy reactance term is of purely an AC nature since it vanishes after time averaging.

\subsection{Power Developed by the AC Sources}

The power performed by the AC potential can be calculated as $P_{A C}(t)=-\left\langle\frac{\partial H_{S}}{\partial t}\right\rangle$, which is

$$
P_{A C}(t)=-\sum_{i} \frac{d V_{i}(t)}{d t}\left\langle d_{i}^{\dagger}(t) d_{i}(t)\right\rangle=\sum_{i} \frac{d V_{i}(t)}{d t} \operatorname{Im}\left\{G_{i, i}^{<}(t, t)\right\} .
$$

Now, replacing Equation (34) in the above equation, and using the Floquet representation, we get

$$
P_{A C}(t)=\sum_{i} \sum_{\alpha=L, R} \sum_{l, m, n} \int \frac{d \varepsilon}{h} n \hbar \omega f_{\alpha}(\varepsilon) \Gamma_{\alpha} \operatorname{Im}\left\{V_{i}(n) \mathcal{G}_{i, l_{\alpha}}(m+l, \varepsilon) \mathcal{G}_{i, l_{\alpha}}^{*}(l, \varepsilon) e^{-i \omega t(m-n)}\right\} .
$$

\section{Relation to the Scattering Matrix Formalism}

In this section, we show that the definition of the heat current in Equation (23), which includes the contribution $J_{c \alpha}^{E}$ due to the contact $\alpha$, is fully in agreement with the scattering matrix formalism. From Equations (43), (45) and (48), the heat reads

$$
\begin{aligned}
\dot{Q}_{\alpha}(t) \quad & =\sum_{l} \int \frac{d \varepsilon}{h} e^{-i l \omega t} \Gamma_{\alpha}\left\{\mathcal{G}_{l_{\alpha}, l_{\alpha}}^{*}(-l, \varepsilon)\left(\varepsilon-\frac{i \hbar \omega}{2}-\mu_{\alpha}\right)\left(f_{\alpha}(\varepsilon)-f_{\alpha}(\varepsilon-l \hbar \omega)\right)\right. \\
& \left.-\sum_{n} \sum_{\beta=L, R}\left(\varepsilon+\frac{l \hbar \omega}{2}-\mu_{\alpha}\right)\left(f_{\alpha}(\varepsilon)-f_{\beta}(\varepsilon-n \hbar \omega)\right) \Gamma_{\beta} \mathcal{G}_{l_{\alpha}, l_{\beta}}(l+n, \varepsilon-n \hbar \omega) \mathcal{G}_{l_{\alpha}, l_{\beta}}^{*}(n, \varepsilon-n \hbar \omega)\right\}
\end{aligned}
$$

Within the scattering matrix approach, the heat flux entering the reservoir $\alpha$ reads $[75,83]$

$$
\dot{Q}_{\alpha}^{S}(t)=\sum_{l, n} e^{-i l \omega t} \int \frac{d \varepsilon}{h}\left(\varepsilon+\frac{l \hbar \omega}{2}-\mu_{\alpha}\right) \sum_{\beta=L, R}\left(f_{\beta}\left(\varepsilon_{-n}\right)-f_{\alpha}(\varepsilon)\right) S_{\alpha \beta}^{*}\left(\varepsilon, \varepsilon_{-n}\right) S_{\alpha, \beta}\left(\varepsilon_{l}, \varepsilon_{-n}\right),
$$

where $\varepsilon_{n}=\varepsilon+n \hbar \omega$ and $S\left(\varepsilon_{n}, \varepsilon_{l}\right)$ is the Floquet scattering matrix which is related to the Green function via the generalized Fisher-Lee relation [68]

$$
S_{\alpha \beta}\left(\varepsilon_{m}, \varepsilon_{n}\right)=\delta_{\alpha, \beta} \delta_{m, n}-i \sqrt{\Gamma_{\alpha}\left(\varepsilon_{m}\right) \Gamma_{\beta}\left(\varepsilon_{n}\right)} \mathcal{G}_{l_{\alpha}, l_{\beta}}\left(m-n, \varepsilon_{n}\right) .
$$

Using this relation, we find that

$$
\begin{aligned}
\dot{Q}_{\alpha}^{S}(t)= & \sum_{l, n} \int \frac{d \varepsilon}{h} e^{-i l \omega t}\left(\varepsilon+\frac{l \hbar \omega}{2}-\mu_{\alpha}\right) \sum_{\beta=L, R}\left(f_{\beta}(\varepsilon-n \hbar \omega)-f_{\alpha}(\varepsilon)\right) \\
& \times \mathcal{G}_{l_{\alpha}, l_{\beta}}^{*}(n, \varepsilon-n \hbar \omega)\left\{i \delta_{\alpha \beta} \delta_{l,-n} \sqrt{\Gamma_{\alpha} \Gamma_{\beta}}+\Gamma_{\alpha} \Gamma_{\beta} \mathcal{G}_{l_{\alpha}, l_{\beta}}(l+n, \varepsilon-n \hbar \omega)\right\},
\end{aligned}
$$

Then, after some algebra and by comparing with Equation (52), we have $\dot{Q}_{\alpha}^{S}(t)=\dot{Q}_{\alpha}(t)$.

\section{Low Frequency Expansion}

For low frequencies of the driving potentials, a solution of the Dyson equation (37) up to $\mathcal{O}(\omega)$ can be obtained by expanding in powers of the AC frequency as

$$
\hat{G}^{R}(t, \varepsilon) \sim \hat{G}^{0}(\varepsilon)+\hat{G}^{R}(t, \varepsilon) \hat{V}(t) \hat{G}^{0}(\varepsilon)+i \hbar \partial_{\varepsilon} \hat{G}^{R}(t, \varepsilon) \frac{d \hat{V}(t)}{d t} \hat{G}^{0}(\varepsilon) .
$$


Now, we can define the frozen Green's function

$$
\hat{G}_{f}^{R}(t, \varepsilon)=\left[\hat{G}^{0}(\varepsilon)^{-1}-\hat{V}(t)\right]^{-1},
$$

in terms of which the exact solution of Equation (37) up to first order in $\omega$ reads

$$
\hat{G}^{R}(t, \varepsilon)=\hat{G}_{f}^{R}(t, \varepsilon)+\frac{i \hbar}{2}\left(\frac{\partial^{2}}{\partial t \partial \varepsilon} \hat{G}_{f}^{R}(t, \varepsilon)+\hat{A}(t, \varepsilon)\right)
$$

with

$$
\hat{A}(t, \varepsilon)=\partial_{\varepsilon} \hat{G}_{f}^{R}(t, \varepsilon) \hat{V}(t) \hat{G}_{f}^{R}(t, \varepsilon)-\hat{G}_{f}^{R}(t, \varepsilon) \hat{V}(t) \partial_{\varepsilon} \hat{G}_{f}^{R}(t, \varepsilon) .
$$

Note that this expression is valid to lowest order in $\omega$, but can be used for arbitrary values of the amplitude $V_{A C}[28,68]$. This is specially appealing if one is interested in addressing nonlinear effects $[65,84,85]$. The validity of the adiabatic approximation considered here was confirmed in [86] by a comparison with an exact calculation in the limit of low level occupations.

We can also expand the Floquet components $\hat{\mathcal{G}}(n, \varepsilon)$ up to first order in $\omega$ as

$$
\hat{\mathcal{G}}(n, \varepsilon) \sim \hat{\mathcal{G}}^{(0)}(n, \varepsilon)+\hbar \omega \hat{\mathcal{G}}^{(1)}(n, \varepsilon),
$$

Then, using the representation of Equation (36) in Equation (58), it is possible to identify the first and second terms of the expansion:

$$
\begin{aligned}
\hat{\mathcal{G}}^{(0)}(n, \varepsilon) & =\int_{0}^{\tau} \frac{d t}{\tau} \hat{G}_{f}^{R}(t, \varepsilon) e^{i n \omega t} \\
\omega \hat{\mathcal{G}}^{(1)}(n, \varepsilon) & =\int_{0}^{\tau} \frac{d t}{\tau} \frac{i}{2}\left(\frac{\partial^{2}}{\partial t \partial \varepsilon} \hat{G}_{f}^{R}(t, \varepsilon)+\hat{A}(t, \varepsilon)\right) e^{i n \omega t} .
\end{aligned}
$$

\section{Application}

We now illustrate our main ideas with a simple but generic example: a single driven level coupled to two fermionic baths kept at the same temperature, but with different chemical potentials, $\mu_{L}=\mu$ and $\mu_{R}=\mu-\delta \mu$. The Hamiltonian of the sample is then

$$
\mathcal{H}_{S}=\varepsilon_{d}(t) d^{+} d
$$

where the level is periodically driven with a monochromatic potential of frequency $\omega$ and amplitude $V_{\mathrm{AC}}, \varepsilon_{d}(t)=\varepsilon_{0}+V(t)$ with $V(t)=V_{\mathrm{AC}} \cos \omega t$. The response of the system will be periodic. Another possibility is to explore heat dynamics in the transient regime, usually in quantum levels whose potential is suddenly changed by an externally-applied voltage pulse. Thermoelectric efficiency can thus be enhanced by a suitable time-dependent potential applied to a quantum dot level far from equilibrium [32,87]. Based on a nonequilibrium Green's function approach, [88] investigates the transient heat current using a complex absorbing potential, greatly reducing the complex calculation of the heat current through a molecule connected to metallic leads and under a gate voltage pulse. Indeed, the transient heat current of a quantum dot in response to a voltage shift can reveal signatures of electronic interaction despite the inherent Coulomb repulsion [89]. The full counting statistics of the quantum energy flow can also be determined for the transient regime, showing universal scaling laws in the single quantum dot case [90]. When the system consists of two levels coupled to a thermal bath, the transient dynamics is sensitive to the employed theoretical formalism (master equation approach or functional influence method) [71].

Here, we restrict ourselves to mean currents. However, their fluctuations can provide us with useful information. For instance, fluctuations of heat beyond the level dictated by the Callen-Welton fluctuation-dissipation theorem signal the presence of backscattering [41]. Furthermore, the energy 
noise reveals additional information not contained in the charge current fluctuations [42]. The novel features are related to electron-hole correlations arising in phase-coherent small conductors driven by periodic voltage biases. The mixed noise between correlations of charge and energy currents shows both interference and transport contributions, and is unique as compared with either charge or energy noises [43-47].

\subsection{Adiabatic Regime and Linear Response in the Bias Voltage}

For small bias voltage, it is possible to combine the procedure described in Section 8 with an expansion in $\delta \mu$. To calculate up to second order in $\omega$ and $\delta \mu$ the heat current of Equation (52), the AC power in Equation (51), and the power developed by the batteries in Equation (20), we need to perform an expansion of the Fermi functions entering the integrals as:

$$
f_{\alpha}(\varepsilon+n \hbar \omega) \simeq f_{\alpha}(\varepsilon)+\partial_{\varepsilon} f_{\alpha} n \hbar \omega+\partial_{\varepsilon}^{2} f_{\alpha} \frac{(n \hbar \omega)^{2}}{2},
$$

with

$$
\begin{aligned}
f_{L}(\varepsilon) & \equiv f(\varepsilon) \\
f_{R}(\varepsilon) & \simeq f(\varepsilon)+\partial_{\varepsilon} f \delta \mu+\partial_{\varepsilon}^{2} f \frac{\delta \mu^{2}}{2} .
\end{aligned}
$$

Accordingly, we can compute all of the fluxes entering Equation (25). Then, up to second order in $\omega$ and $\delta \mu$, the total dissipative power $P_{\text {tot }}^{\text {diss }}$ reads:

$$
P_{\mathrm{tot}}^{\mathrm{diss}}(t)=L^{\delta \mu^{2}}(t) \delta \mu^{2}+L^{\omega^{2}}(t) \omega^{2},
$$

where the transport coefficients are:

$$
\begin{aligned}
L^{\delta \mu^{2}}(t) & =\int \frac{d \varepsilon}{h} \partial_{\varepsilon} f \Gamma_{L} \Gamma_{R}\left|G_{f}^{R}(t, \varepsilon)\right|^{2} \\
L^{\omega^{2}}(t) & =\frac{\hbar}{2 \omega^{2}} \int \frac{d \varepsilon}{2 \pi} \partial_{\varepsilon} f \Gamma^{2}\left|\partial_{t} G_{f}^{R}(t, \varepsilon)\right|^{2},
\end{aligned}
$$

where $\Gamma=\Gamma_{L}+\Gamma_{R}$ is the total hybridization. Notice that, as is shown is [33] for systems with time-reversal symmetry, the terms that are proportional to $\omega \delta \mu$ do not contribute to the total dissipated power.

Following a similar procedure in Equation (52) and then subtracting Equation (66), we find that the dissipated heat flux of the central piece is

$$
\dot{Q}_{S}^{\text {diss }}(t)=L_{d}^{\omega}(t) \omega+L_{d}^{\omega \delta \mu}(t) \omega \delta \mu+L_{d}^{\omega^{2}}(t) \omega^{2}
$$

with

$$
\begin{aligned}
& L_{d}^{\omega}(t)=-\int \frac{d \varepsilon}{2 \pi} \partial_{\varepsilon} f(\varepsilon-\mu) \Gamma\left|G_{f}^{R}(t, \varepsilon)\right|^{2} V_{\mathrm{ac}} \sin (\omega t), \\
& L_{d}^{\omega \delta \mu}(t)=\int \frac{d \varepsilon}{2 \pi} \Gamma_{R} \partial_{\varepsilon} f(\varepsilon-\mu) \partial_{\varepsilon}\left|G_{f}^{R}(t, \varepsilon)\right|^{2} V_{\mathrm{AC}} \sin (\omega t), \\
& L_{d}^{\omega^{2}}(t)=\frac{\hbar}{2 \omega^{2}} \int \frac{d \varepsilon}{2 \pi} \Gamma^{2} \partial_{\varepsilon} f(\varepsilon-\mu) \partial_{t}\left(\partial_{\varepsilon} G_{f}^{R}(t, \varepsilon) \partial_{t} G_{f}^{R^{*}}(t, \varepsilon)\right) .
\end{aligned}
$$


In Figure 3 (upper panel) we show the total heat generated in the reservoirs as a function of time for zero temperature (red dashed line) and nonzero temperature (blue line). In the latter case, an individual heat flux can become negative but the total dissipated heat must be positive for all temperatures, as shown in Figure 3 (lower panel). Note that one should also take into account the contribution from the sample, which is purely dynamical (Figure 3 (middle panel)) and vanishes for zero temperature.

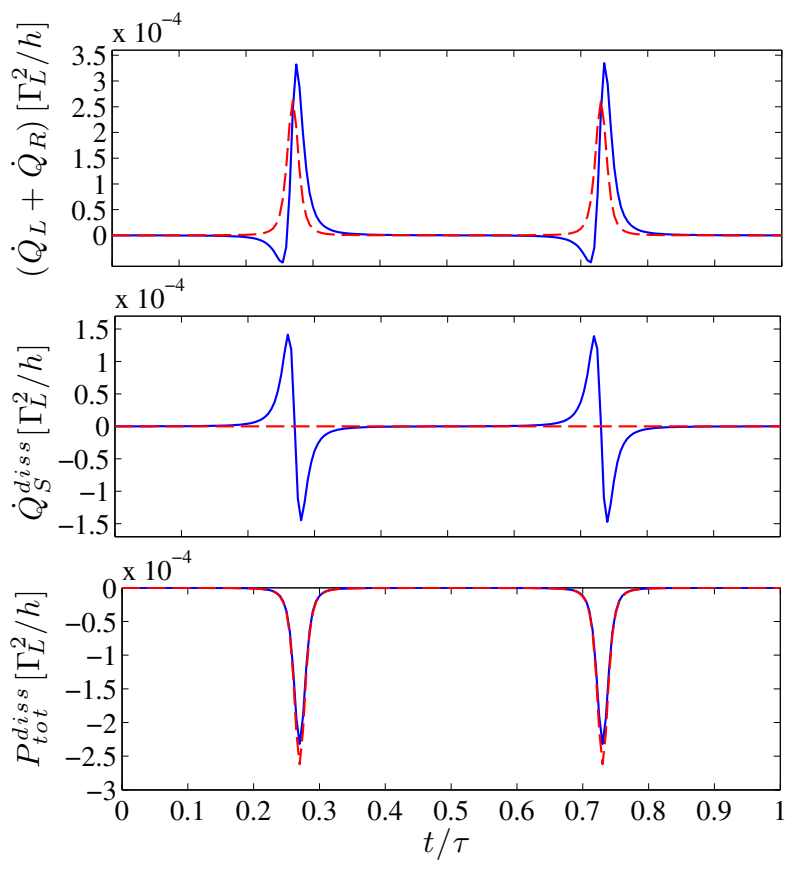

Figure 3. Adiabatic regime of a single driven quantum level coupled to two fermionic baths. The different components of the total heat production $Q_{\text {tot }}^{\text {diss }}(t)=-P_{\text {tot }}^{\text {diss }}(t)$ as a function of time. Dashed lines correspond to reservoirs at $T=0$, while solid lines are for $k_{B} T=0.02$. Energies are expressed in units of $\Gamma_{L}$. The energy of the level evolves in time with $V_{A C}=-8$ and $\hbar \omega=1 \times 10^{-3}$. Parameters: $\mu=1, \delta \mu=0.004, \varepsilon_{0}=0$ and hybridization widths $\Gamma_{L}=1$ and $\Gamma_{R}=0.5$.

\subsection{Nonadiabatic Regime}

We now consider the entropy production for high driving frequencies, where the linear response approach of Section 9.1 is not applicable. The evaluation of the currents implies the evaluation of the exact Dyson equation (37) following the methods of [66,67]. A similar situation was analyzed in [91], where it was found that the rate of change for the nonadiabatic energy agrees with the work done on a molecule subjected to a time-dependent applied field. The work in [92] discusses heat transfer across a triple dot structure with the nonadiabatic driving applied to an edge dot.

In our case, we focus on reservoirs at zero temperature for simplicity. We see in the figure that, unlike the adiabatic case, the heat flux at the reservoirs may attain negative values for short (but finite) time intervals (see Figure 4 (upper panel)). Nevertheless, we emphasize that the total entropy production is always positive at every instant (see Figure 4 (lower panel)). For this to occur the dissipated heat at the sample must be nonvanishing and positive (see Figure 4 (middle panel)). 

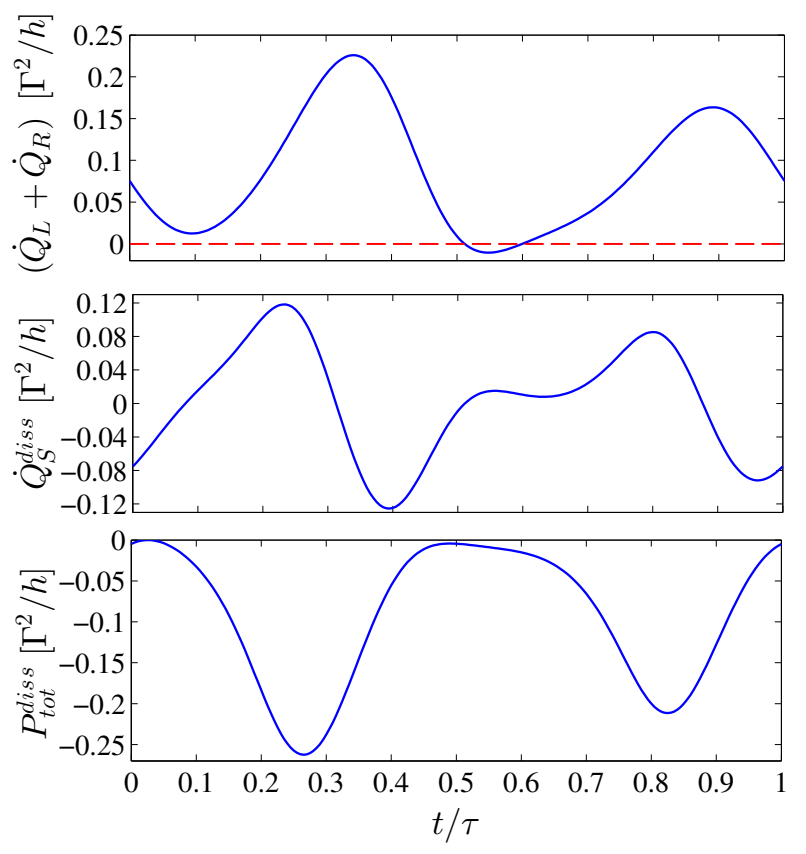

Figure 4. Nonadiabatic regime of a single driven quantum level coupled to two fermionic baths at $T=0$. The different components of the total heat production $Q_{\mathrm{tot}}^{\text {diss }}(t)=-P_{\text {tot }}^{\text {diss }}(t)$ as a function of time. The energy of the level evolves in time with $V_{A C}=0.7$ and $\hbar \omega=0.3$. Parameters: $\mu=0.2, \delta \mu=0, \varepsilon_{0}=0$, and hybridization widths $\Gamma_{L}=\Gamma_{R}=0.5$. Energies are expressed in units of $\Gamma=\Gamma_{L}+\Gamma_{R}$. The upper panel shows that the heat flux at the reservoirs may instantaneously attain negative values. The dissipative heat flux at the driven $\operatorname{dot} Q_{S}^{\text {diss }}(t)$ is highly fluctuating but the sum of the two contributions satisfies $Q_{\text {tot }}^{\text {diss }}(t) \geq 0$, consistent with the second law.

\section{Conclusions}

The subject of time-dependent heat in quantum electronic systems presents unique aspects as compared with the better understood macroscopic case. In this work, we have briefly reviewed the recent developments in the field. On the basis of general thermodynamic arguments and conservation laws we have presented exact equations for the time-resolved energy conversion in a quantum system in contact with AC driving sources and reservoirs at which DC bias voltages are applied. These equations lead to the unambiguous definition of the total heat and production in the full system containing the central driven device, the reservoirs and the contacts between the different parts. These results are independent of the particular method used to evaluate the physical quantities involved. In order to get the exact solution of an example we have used, for convenience, a Green function technique as in $[52,53]$. Of course, the physical picture is beyond the method employed to solve the particular problem.

We have also analyzed the possibility of defining the heat flows not only resolved in time but also in space, in the sense of identifying flows in the reservoirs and through the piece that is in contact with the AC sources. How to properly perform this split in a physically-meaningful way is a non-trivial task. Intuitively, we expect that deep in the reservoirs, we should have an equilibrium system. For adiabatically-driven systems, we have found that this seems to be, indeed the case. Then, by suitably generalizing the definition of the time-dependent heat current. We argue that the proper generalization is given by Equation (23), which implies taking into account the rate of change of the energy stored at the reservoir plus half of the one stored at the contact. Such a definition leads to results compatible with scattering matrix expressions for continuum models, and to the derivation of a Joule law when the reservoirs are at zero temperature.

However, for highly non-equilibrium driving at the central piece and strongly-coupled reservoirs, it is not obvious at all if we should ever recover an equilibrium state at every instant, even deep 
inside the reservoirs. In fact, we have analyzed the example of a single-level system driven by a high-frequency gate voltage in contact with reservoirs at zero temperature and we have found that the definition of the instantaneous heat current at the reservoirs (Equation (23)) that leads to meaningful physical results in the adiabatic regime may be negative at some instants, meaning a temporary flow of heat exiting the reservoirs. This result is accompanied by a finite value of what we have identified as the heat flow in the central piece. The sum of both contributions leads to a net instantaneous positive value of the total entropy production. Hence, the result is in agreement with the second law, albeit non-intuitive. This is because the natural expectation is to have a dissipative energy flow only into the reservoirs and not in the central device. This is, in fact, the case when we consider the average over one period of the driving. However, instantaneously, and for non-equilibrium situations, the exact equation for the entropy production is given by Equation (25), which contains contributions associated to the reservoirs, contacts and the central device. The second law implies that the sum of all these contributions should be positive but no fundamental law imposes independent conditions on the individual terms. Finally, we have commented on the difficulties of accessing experimentally a reliable test of the time- and spatially-resolved heat transport discussed in the present work.

Acknowledgments: This work was supported by MINECO under Grant No. FIS2014-52564, UBACyT, CONICET and MINCyT, Argentina.

Author Contributions: All the authors conceived the theoretical model and performed the calculations. They analyzed the results and wrote the paper. All the authors read and approved the final manuscript.

Conflicts of Interest: The authors declare no conflict of interest.

\section{References}

1. Benenti, G.; Casati, G.; Saito, K.; Whitney, R.S. Fundamental aspects of steady-state conversion of heat to work at the nanoscale. arXiv 2016, arXiv:1608.05595.

2. Kosloff, R. Quantum thermodynamics: A dynamical viewpoint. Entropy 2013, 15, 2100-2128.

3. Campisi, M.; Fazio, R.J. Dissipation, Correlation and Lags in Heat Engines. Phys. A Math. Theor. 2016, 49,345002 .

4. Gasparinetti, S.; Solinas, P.; Pugnetti, S.; Fazio, R.; Pekola, J.P. Environment-governed dynamics in driven quantum systems. Phys. Rev. Lett. 2013, 110, 150403.

5. Segal, D. Heat transfer in the spin-boson model: A comparative study in the incoherent tunneling regime. Phys. Rev. E 2014, 90, 012148.

6. Carrega, M.; Solinas, P.; Sassetti, M.; Weiss, U. Energy Exchange in Driven Open Quantum Systems at Strong Coupling. Phys. Rev. Lett. 2016, 116, 240403.

7. Schmidt, R.; Carusela, M.F.; Pekola, J.P.; Suomela, S.; Ankerhold, J. Work and heat for two-level systems in dissipative environments: Strong driving and non-Markovian dynamics. Phys. Rev. B 2015, 91, 224303.

8. Ponomarev, A.V.; Denisov, S.; Hänggi, P. AC-Driven Atomic Quantum Motor. Phys. Rev. Lett. 2009, $102,230601$.

9. Abah, O.; Roßnagel, J.; Jacob, G.; Deffner, S.; Schmidt- Kaler, F.; Singer, K.; Lutz, E. Single-ion heat engine at maximum power. Phys. Rev. Lett. 2012, 109, 203006.

10. Brantut, J.-P.; Grenier, C.; Meineke, J.; Stadler, D.; Krinner, S.; Kollath, C.; Esslinger, T.; Georges, A. A thermoelectric heat engine with ultracold atoms. Science 2013, 342, 713-715.

11. Uzdin, R.; Levy, A.; Kosloff, R. Equivalence of quan- tum heat machines, and quantum-thermodynamic signatures. Phys. Rev. X 2015, 5, 031044.

12. Blickle, V.; Bechinger, C. Realization of a micrometer-sized stochastic heat engine. Nat. Phys. 2011, 8, 143-146.

13. Martínez, I.A.; Roldán, E.; Dinis, L.; Petrov, D.; Rica, R.A. Adiabatic Processes Realized with a Trapped Brownian Particle. Phys. Rev. Lett. 2015, 114, 120601.

14. Scully, M.O. Quantum photocell: Using quantum co-herence to reduce radiative recombination and increase efficiency. Phys. Rev. Lett. 2010, 104, 207701.

15. Steeneken, P.G.; Le Phan, K.; Goossens, M.J.; Koops, G.E.J;; Brom, G.J.A.M.; van der Avoort, C.; van Beek, J.T.M. Piezoresistive heat engine and refrigerator. Nat. Phys. 2010, 7, 354-359. 
16. Scully, M.O.; Zubairy, M.S.; Agarwal, G.S.; Walther, H. Extracting work from a single heat bath via vanishing quantum coherence. Science 2003, 299, 862-864.

17. Chamon, C.; Mucciolo, E.R.; Arrachea, L.; Capaz, R.B. Heat Pumping in Nanomechanical Systems. Phys. Rev. Lett. 2011, 106, 135504.

18. Arrachea, L.; Mucciolo, E.R.; Chamon, C.; Capaz, R.B. Microscopic model of a phononic refrigerator. Phys. Rev. B 2012, 86, 125424.

19. Steele, G.A.; Ḧ̈tel, A.K.; Witkamp, B.; Poot, M.; Meerwaldt, H.B.; Kouwenhoven, L.P.; van der Zant, H.S.J. Strong coupling between single-electron tunneling and nanomechanical motion. Science 2009, 325, 1103-1107.

20. Lotze, C.; Corso, M.; Franke, K.J.; von Oppen, F.; Pascual, J.I. Driving a macroscopic oscillator with the stochastic motion of a hydrogen molecule. Science 2012, 338, 779-782.

21. Brandner, K.; Saito, K.; Seifert, U. Thermodynamics of micro- and nano-systems driven by periodic temperature variations. Phys. Rev. X 2015, 5, 031019.

22. Horowitz, J.M. Quantum-trajectory approach to the stochastic thermodynamics of a forced harmonic oscillator. Phys. Rev. E 2012, 85, 031110.

23. Martinez, E.A.; Paz, J.P. Dynamics and thermodynamics of linear quantum open systems. Phys. Rev. Lett. 2013, 110, 130406.

24. Freitas, N.; Paz, J.P. Fundamental limits for cooling of linear quantum refrigerators. arXiv 2016, arXiv:1607.04234.

25. Arrachea, L.; Moskalets, M.; Martin-Moreno, L. Heat production and energy balance in nanoscale engines driven by time-dependent fields. Phys. Rev. B 2007, 75, 245420.

26. Rey, M.; Strass, M.; Kohler, S.; Hänggi, P.; Sols, F. Nonadiabatic electron heat pump. Phys. Rev. B 2007, $76,08533$.

27. Juergens, S.; Haupt, F.; Moskalets, M.; Splettstoesser, J. Thermoelectric performance of a driven double quantum dot. Phys. Rev. B 2013, 87, 245423.

28. Moskalets, M.; Büttiker, M. Heat production and current noise for single- and double-cavity quantum capacitors. Phys. Rev. B 2009, 80, 081302.

29. Bustos-Marun, R.; Refael, G.; von Oppen, F. Adiabatic Quantum Motors. Phys. Rev. Lett. 2013, 111, 060802.

30. Arrachea, L.; von Oppen, F. Nanomagnet coupled to quantum spin Hall edge: An adiabatic quantum motor. Physica E 2015, 74, 596-602.

31. Daré, A.M.; Lombardo, P. Time-dependent thermoelectric transport for nanoscale thermal machines. Phys. Rev. B 2016, 93, 035303.

32. Zhou, H.; Thingna, J.; Hänggi, P.; Wang, J.-S.; Li, B. Boosting thermoelectric efficiency using time-dependent control. Sci. Rep. 2015, 5, 14870.

33. Ludovico, M.F.; Battista, F.; von Oppen, F.; Arrachea, L. Adiabatic response and quantum thermoelectrics for AC-driven quantum systems. Phys. Rev. B 2016, 93, 075136.

34. Bergenfeldt, C.; Samuelsson, P.; Sothmann, B.; Flindt, C.; Büttiker, M. Hybrid microwave-cavity heat engine. Phys. Rev. Lett. 2014, 112, 076803.

35. Biele, R.; D'Agosta, R.; Rubio, A. Time-dependent thermal transport theory. Phys. Rev. Lett. 2015, 115, 056801.

36. Alicki, R.; Gelbwaser-Klimovsky, D.; Szczygielski, K. Solar cell as self-oscillating heat engine. J. Phys. A Math. Theor. 2016, 49, 015002.

37. Alicki, R. Thermoelectric generators as self-oscillating heat engines. J. Phys. A Math. Theor. 2016, 49, 085001.

38. Kindermann, M.; Pilgram, S. Statistics of heat transfer in mesoscopic circuits. Phys. Rev. B 2004, 69, 155334.

39. Averin, D.V.; Pekola, J.P. Violation of the Fluctuation-Dissipation Theorem in Time-Dependent Mesoscopic Heat Transport. Phys. Rev. Lett. 2010, 104, 220601.

40. Sergi, D. Energy transport and fluctuations in small conductors. Phys. Rev. B 2011, 83, 033401.

41. Moskalets, M. Floquet scattering matrix theory of heat fluctuations in dynamical quantum conductors. Phys. Rev. Lett. 2014, 112, 206801.

42. Battista, F.; Haupt, F.; Splettstoesser, J. Energy and power fluctuations in AC-driven coherent conductors. Phys. Rev. B 2014, 90, 085418.

43. Sánchez, R.; Sothmann, B.; Jordan, A.N.; Büttiker, M. Correlations of heat and charge currents in quantum-dot thermoelectric engines. New J. Phys. 2013, 15, 125001.

44. Battista, F.; Haupt, F.; Splettstoesser, J. Correlations between charge and energy current in AC-driven coherent conductors. J. Phys. Conf. Ser. 2014, 568, 052008. 
45. Crépieux, A.; Michelini, F. Mixed, charge and heat noises in thermoeleectric nanosystems. J. Phys. Condens. Matter 2015, 27, 015302.

46. Crépieux, A.; Michelini, F. Heat-charge mixed noise and thermoelectric efficiency fluctuations. J. Stat. Mech. arXiv 2016, arXiv:1510.01056.

47. Eyméoud, P.; Crépieux, A. Mixed electrical-heat noise spectrum in a quantum dot. arXiv 2016, arXiv:1608.02414.

48. Campisi, M.; Pekola, J.; Fazio, R. Nonequilibrium fluctuations in quantum heat engines: Theory, example, and possible. New J. Phys. 2015, 17, 035012.

49. Caso, A.; Arrachea, L.; Lozano, G.S. Local temperatures and heat flow in quantum driven systems. Phys. Rev. B 2011, 83, 165419.

50. Arrachea, L.; Cugliandolo, L.F. Study of a fluctuation-dissipation relation of a dissipative driven mesoscopic system. EPL Europhys. Lett. 2005, 70, 642-648.

51. Caso, A.; Arrachea, L.; Lozano, G.S. Local and effective temperatures of quantum driven systems. Phys. Rev. B 2010, 81, 041301.

52. Ludovico, M.F.; Lim, J.S.; Moskalets, M.; Arrachea, L.; Sánchez, D. Dynamical energy transfer in AC-driven quantum systems. Phys. Rev. B 2014, 89, 161306.

53. Ludovico, M.F.; Moskalets, M.; Sánchez, D.; Arrachea, L. Dynamics of energy transport and entropy production in AC-driven quantum electron systems. Phys. Rev. B 2016, 94, 035436.

54. Moskalets, M.; Haack, G. Heat and charge transport measurements to access single-electron quantum characteristics. arXiv 2016, arXiv:1609.04544.

55. Ludovico, M.F.; Lim, J.S.; Moskalets, M.; Arrachea, L.; Sánchez, D. Time resolved heat exchange in driven quantum systems. J. Phys. Conf. Ser. 2014, 568, 052017.

56. Büttiker, M.; Prêtre, A.; Thomas, H. Mesoscopic capacitors. Phys. Lett. A 1993, 180, 364-369.

57. Brouwer, P.W. Scattering approach to parametric pumping. Phys. Rev. B 1998, 58, 10135.

58. Avron, J.E.; Elgart, A.; Graf, G.M.; Sadum, L. Optimal Quantum Pumps. Phys. Rev. B 2001, 87, 236601.

59. Vavilov, M.G.; Ambegoakar, V.; Aleiner, I.L. Charge pumping and photovoltaic effect in open quantum dots. Phys. Rev. B 2001, 63, 195313.

60. Pastawski, H.M. Classical and quantum transport from generalized Landauer-B̈̈tiker equations II. Time-dependent resonant tunneling. Phys. Rev. B 1992, 63, 4053-4070.

61. Jauho, A.-P.; Wingreen, N.S.; Meir, Y. Time-dependent transport in interacting and noninteracting resonant-tunneling systems. Phys. Rev. B 1994, 50, 5528.

62. Platero, G.; Aguado, R. Photon-assisted transport in semiconductor nanostructures. Phys. Rep. 2004, 395, 1-157.

63. Kohler, S.; Lehmann, J.; Hänggi, P. Driven quantum transport on the nanoscale. Phys. Rep. 2005, 406, $379-443$.

64. Moskalets, M. Scattering Matrix Approach to Non-Stationary Quantum Transport; Imperial College Press: London, UK, 2011.

65. Moskalets, M.; Samuelsson, P.; Büttiker, M. Quantized Dynamics of a Coherent Capacitor. Phys. Rev. B 2008, 100, 086601.

66. Arrachea, L. Green-function approach to transport phenomena in quantum pumps. Phys. Rev. B 2005 72,125349 .

67. Arrachea, L. Exact Green's function renormalization approach to spectral properties of open quantum systems driven by harmonically time-dependent fields. Phys. Rev. B 2007, 75, 035319.

68. Arrachea, L.; Moskalets, M. Relation between scattering-matrix and Keldysh formalisms for quantum transport driven by time-periodic fields. Phys. Rev. B 2006,74, 245322.

69. Bruch, A.; Thomas, M.; Kusminskiy, S.V.; von Oppen, F.; Nitzan, A. Quantum thermodynamics of the driven resonant level model. Phys. Rev. B 2016, 93, 115318.

70. Ochoa, M.A.; Bruch, A.; Nitzan, A. Energy distribution and local fluctuations in strongly coupled open quantum systems: The extended resonant level model. Phys. Rev. B 2016, 94, 035420.

71. Carrega, M.; Solinas, P.; Braggio, A.; Sassetti, M. Computation of transient dynamics of energy power for a dissipative two state system. J. Stat. Mech. 2016, 054014.

72. Carrega, M.; Solinas, P.; Braggio, A.; Sassetti, M.; Weiss, U. Functional integral approach to time-dependent heat exchange in open quantum systems: General method and applications. New J. Phys. 2015, 17, 045030.

73. Büttiker, M.; Thomas, H.; Prêtre, A. Current partitioning in multiprobe conductors in the presence of slowly oscillating external potentials. Z. Phys. B 1994, 94, 133-137. 
74. Chen, J.; ShangGuan, M.; Wang, J. A gauge invariant theory for time dependent heat current. New J. Phys. 2015, 17, 053034.

75. Lim, J.S.; López, R.; Sánchez, D. Dynamic thermoelectric and heat transport in mesoscopic capacitors. Phys. Rev. B 2013, 88, 201304.

76. Lv, Y.; Chen, J.; Zhen, R.-K.; Song, J.; Zhang, T.; Li, X.; Shi, X.; Chen, L. Photo-induced enhancement of the power factor of $\mathrm{Cu}_{2} \mathrm{~S}$ thermoelectric films. Sci. Rep. 2015, 5, 16291.

77. Proesmans, K.; Cleuren, B.; Van den Broeck, C. Linear stochastic thermodynamics for periodically driven systems. J. Stat. Mech. 2016, 023202.

78. García-Cañadas, J.; Min, G. Thermal dynamics of thermoelectric phenomena from frequency resolved methods. AIP Adv. 2016, 6, 035008.

79. Rosselló, G.; Lim, J.S.; López, R. Time-dependent heat flow in interacting quantum conductors. Phys. Rev. B 2015, 92, 115402.

80. Esposito, M.; Ochoa, M.A.; Galperin, M. Quantum thermodynamics: A nonequilibrium Green's function approach. Phys. Rev. Lett. 2015, 114, 080602.

81. Esposito, M.; Ochoa, M.A.; Galperin, M. Nature of heat in strongly coupled open quantum systems. Phys. Rev. B 2015, 92, 235440.

82. Seifert, U. First and second law of thermodynamics at strong coupling. Phys. Rev. Lett. 2016, 116, 020601.

83. Battista, F.; Moskalets, M.; Albert, M.; Samuelsson, P. Quantum heat fluctuations of single-particle sources. Phys. Rev. Lett. 2013, 110, 126602.

84. Kashuba, O.; Schoeller, H.; Splettstoesser, J. Nonlinear adiabatic response of interacting quantum dots. Europhys. Lett. 2012, 98, 57003.

85. Alomar, M.I.; Lim, J.S.; Sánchez, D. Coulomb-blockade effect in nonlinear mesoscopic capacitors. Phys. Rev B 2016, 94, 165425.

86. Nocera, A.; Perroni, C.A.; Ramaglia, V.M.; Cataudella, V. Charge and heat transport in soft nanosystems in the presence of time-dependent perturbations. Beilstein J. Nanotechnol. 2016, 7, 439-464.

87. Crépieux, A.; Simkovic, F.; Cambon, B.; Michelini, F. Enhanced thermopower under a time-dependent gate voltage. Phys. Rev. B 2011, 83, 153417.

88. Yu, Z.; Zhang, L.; Xing, Y.; Wang, J. Investigation of transient heat current from first principles using complex absorbing potential. Phys. Rev. B 2014, 90, 115428.

89. Schulenborg, J.; Saptsov, R.B.; Haupt, F.; Splettstoesser, J.; Wegewijs, M.R. Fermion-parity duality and energy relaxation in interacting open systems. Phys. Rev. B 2016, 93, 081411.

90. Yu, Z.; Tang, G.M.; Wang, J. Full-counting statistics of transient energy current in mesoscopic systems. Phys. Rev. B 2016, 93, 195419.

91. Mandal, A.; Hunt, K.L.C. Non-adiabatic current densities, transitions, and power absorbed by a molecule in a time-dependent electromagnetic field. J. Chem. Phys. 2015, 143, 034102.

92. Gallego-Marcos, F.; Platero, G. Coherent long-range thermoelectrics in nonadiabatic driven quantum systems. arXiv 2016, arXiv:1607.06000.

(C) 2016 by the authors; licensee MDPI, Basel, Switzerland. This article is an open access article distributed under the terms and conditions of the Creative Commons Attribution (CC-BY) license (http://creativecommons.org/licenses/by/4.0/). 